\title{
Imaging of the stroke-related changes in the vascular system of the mouse brain with the use of extended focus Optical Coherence Microscopy
}

\author{
Szymon Tamborski ${ }^{1}$, Hong Chou Lyu ${ }^{1}$, Danuta Bukowska ${ }^{2}$, Hubert Dolezyczek ${ }^{3}$, \\ Grzegorz Wilczynski ${ }^{3}$, Daniel Szlag ${ }^{4}$, Theo Lasser ${ }^{4}$, Maciej Wojtkowski ${ }^{1}$ and Maciej Szkulmowski ${ }^{1}$ \\ ${ }^{1}$ Institute of Physics, Faculty of Physics, Astronomy and Informatics, \\ Nicolaus Copernicus University Grudziadzka 5, 87-100 Torun, Poland \\ ${ }^{2}$ Lions Eye Institute, 2 Verdun Street, Nedlands WA 6009, Australia \\ ${ }^{3}$ Nencki Institute of Experimental Biology, 3 Pasteur Street, 02-093 Warsaw, Poland \\ ${ }^{4}$ École Polytechnique Fédérale de Lausanne, CE 3 316, Station 1, CH-1015 Lausanne, Switzerland
}

\begin{abstract}
We used Optical Coherence Microscopy (OCM) to monitor structural and functional changes due to ischemic stroke in small animals brains in vivo. To obtain lateral resolution of $2.2 \mu \mathrm{m}$ over the range of $600 \mu \mathrm{m}$ we used extended focus configuration of OCM instrument involving Bessel beam. It provided access to detailed 3D information about the changes in brain vascular system up to the level of capillaries across I and II/III layers of neocortex. We used photothrombotic stroke model involving photoactive application of rose bengal to assure minimal invasiveness of the procedure and precise localization of the clot distribution center. We present the comparative analysis involving structural and angiographic maps of the stroke-affected brain enabling in-depth insight to the process of development of the disorder.
\end{abstract}

Keywords: optical coherence microscopy, extended focus, Bessel beam, biomedical imaging, rose bengal, photothrombosis, ischemic stroke

\section{INTRODUCTION}

Stroke remains one of the most frequent causes of death and permanent disability worldwide. One in six people will be affected by this injury in their lifetime [1]. It directly and violently corrupts the central nervous system disabling its particular domains. Therefore, its consequences may affect many different parts of the body and fields of mind. Stroke streaks fast which implies the need for immediate precise diagnose and instant treatment of the sufferer. To make it possibly effective and to improve prevention the stroke mechanism should be very well recognized. Here, the core issue is the operation of the brain vascular system, recognition of its defects leading to stroke and the ability to recover after injury. Investigations in this field performed with the use of animal stroke models deliver valuable information which potentially can be translated to the human case [2]. The number of methods are already in use for visualisation of the vascular system: laser speckle angiography, Doppler ultrasound imaging, photoacoustic probing, two photon microscopy, Positron Emission Tomography and Optical Coherence Microscopy (OCM). Among them OCM stands out because of its ability to three-dimensional imaging of the brain tissue with micron-scale resolution of the reasonable range of few millimetres in time scale enabling in vivo measurements [3]. Furthermore, available angiographic OCM measurement protocols making use of speckle decorrelation in time allow for contrasting of blood vessels with the variation of the light signal due to the movement of the scattering red blood cells [4]. The angiographic modalities show great potential to deliver high resolution 3D maps of brain vascular system up to the level of microcapillaries whose dynamical changes in case of stroke can be analysed in time in systematic way.

Whereas in OCM axial resolution is limited by coherence gate width determined by the spectrum width of the light source, the lateral resolution is specified by the numerical aperture of illumination-detection system via classical diffraction principle. Desired high resolution is achieved at the expense of limited depth of focus. To take full advantage

Optical Coherence Tomography and Coherence Domain Optical Methods in Biomedicine XX, edited by Joseph A. Izatt, James G. Fujimoto, Valery V. Tuchin, Proc. of SPIE Vol. 9697,

$969718 \cdot$ ? 2016 SPIE · CCC code: 1605-7422/16/\$18 · doi: 10.1117/12.2214779 
of 3D imaging functionality of OCM and to have high resolution preserved within the in-depth region of interest $(>500$ $\mu \mathrm{m})$ we utilized extended focus setup [5,6]. It works with Bessel beam created with the use of axicon lens and assures the resolution of $2.2 \mu \mathrm{m}$ within the range of $600 \mu \mathrm{m}$.

In our work we decided to use photothrombosis model of ischemic stroke [7]. Here, clot is generated after illuminating the vessel containing phototoxic dye (bengal rose) dissolved in blood with the light of wavelength absorbable by the agent. The model usually is utilized by illuminating the whole accessible area of the brain producing the clots in all exposed vessels. However, we rather focus the photoactivating light on the particular artery. Such local realization of the procedure allows for observation of the dynamics of flow changes in the part of circulation system supplied by the chosen blocked artery and therefore is more appropriate to mimic what occurs in reality in case of stroke. In contrast to methods using mechanical artery occlusions [8], here is no need for animal removal from the imaging space which enables continuous observation of the clot grow and its influence on the flow in the vessels conjugated to the illuminated one.

Here we present the results of stroke-related changes of microvascular system dynamics in mouse brain after local generation of the clot. We show the potential of the multimodal interferometer system combining extended focus Optical Coherence Microscope for both structural and angiographic imaging, the photothrombosis induction subsystem and brightfield microscope for control of the experiment and structural image reference. All experimental procedures involving animals were executed in accordance with the policy of the National Committee of Bioethics for the Affairs of Experiments on Animals.

\section{MATERIALS AND METHODS}

\subsection{Experimental setup}

As announced in the Introduction the experimental setup was designed as the platform for comprehensive structural and functional measurements of the mouse brain in vivo with additional laser beam path for phototoxic stroke induction and bright-field preview. The schematic diagram of the setup is shown in Fig. 1.

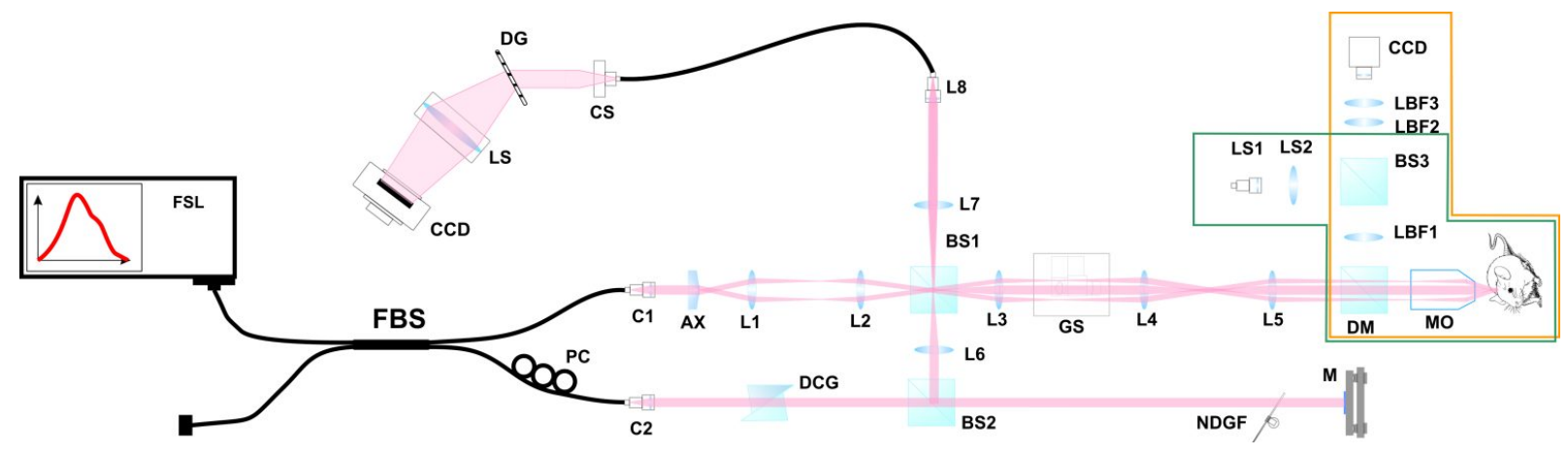

Fig. 1. Experimental setup; FSL - femtosecond Ti:Saph laser, FBS - fiber beamsplitter, PC - polarization controller, C1, C2, CS - collimators, AX - axicon lens, L1-L8, LBF1-LBF3 and LS1-LS2, LS - lenses, BS1-BS3 - beamsplitters, DM dichroic mirror, GS - galvo scanners, MO - microscope objective, M - mirror, DCG - dispersion compensation glass, NDGF - neutral density gradient filter, DG - diffraction grating. Part of the diagram outlined in green - green laser diode path for stroke photoinduction. Part of the diagram outlined in amber - bright field microscope.

As a light source we used femtosecond laser (Fusion, Femtolasers; central wavelength: 795 nm, spectrum FWHM: 130 $\mathrm{nm})$. The interferometer was constructed in Mach-Zehnder scheme. In the illumination path we used the axicon lens (Thorlabs, apex angle $176^{\circ}$ ) to produce Bessel beam which was delivered by two telescopes to the actual microscopic system (objective lens: Olympus 10x, NA 0.3). 

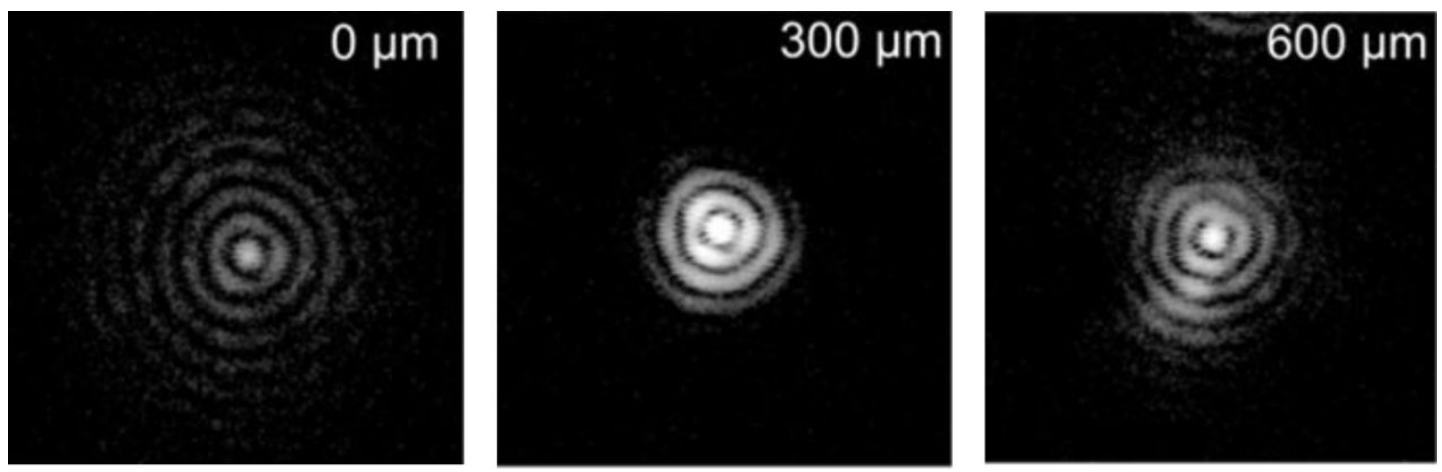

Fig. 2. Lateral cross-sections of the point spread function of the optical setup. The Bessel pattern is visible and the central peak FWHM is preserved along the range of $600 \mu \mathrm{m}$. Values of depth at which scatterers were located are indicated on the picture. The values are given with respect to the arbitrary point on optical axis located close to the zero-optical-pathdifference position. The dimensions of each image are $40 \mu \mathrm{m} \times 40 \mu \mathrm{m}$.

In the proposed geometry the central peak of Bessel spot was confirmed to maintain width of $2.2 \mu \mathrm{m}$ along the path of $600 \mu \mathrm{m}$. This was measured by imaging of the artefact consisting of the separated scattering iron oxide powder particles of size appropriately smaller than the expected resolution $(<200 \mathrm{~nm})$ embedded in an optically clear polyurethane resin (National Physical Laboratory, UK). High spatial oversampling enabled reconstruction of the point spread function of the optical setup and thus determination of the effective resolution as a function of depth. The exemplary lateral cross sections of the point spread function obtained for individual scatterer located at different depths are presented in Fig. 2. One can see that the diameter of the central peak of Bessel-like pattern determining effective resolution maintains constant value of $2.2 \mu \mathrm{m}$. Its detected intensity, however varies along focus due to both - properties of the Bessel beam and the aperture of Gaussian detection.
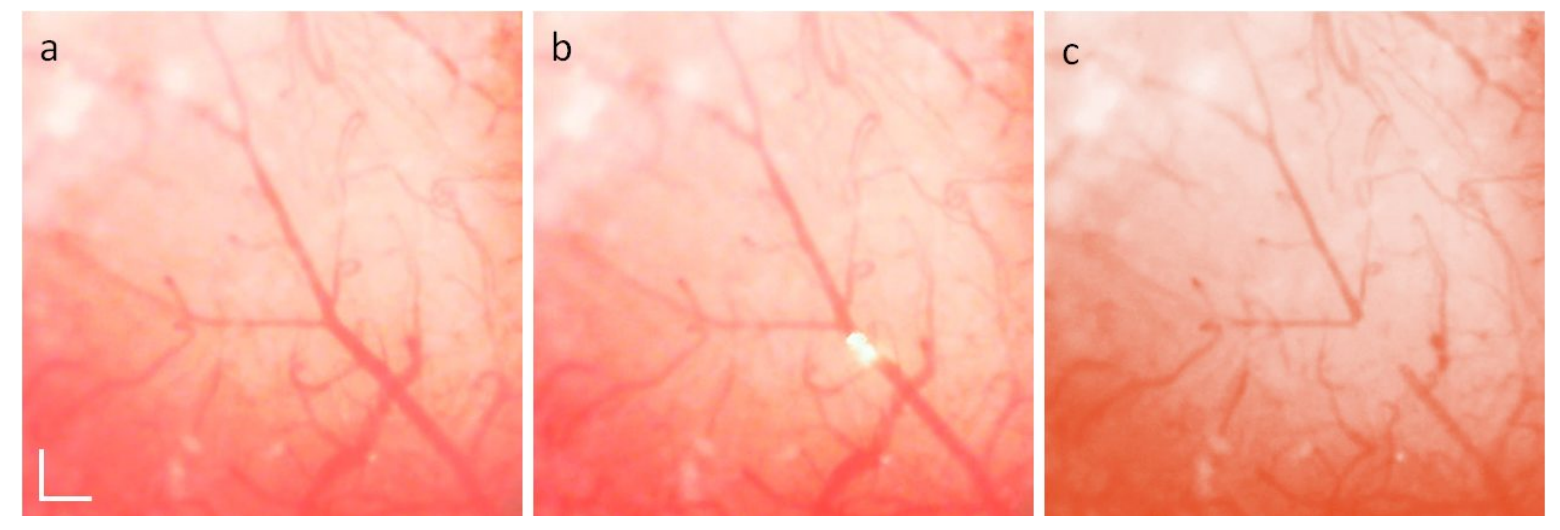

Fig. 3 Images from the brightfield microscopic preview presenting the clot formation due to photothrombosis; a) original vasculature of the animals' brain, b) $520 \mathrm{~nm}$ laser diode spot targeted at a big artery, c) the vasculature defects after main clot formation in the targeted spot. Scale bar: $100 \mu \mathrm{m}$.

The beam was scanned across the sample with the use of a pair of galvo scanners. The microscope worked in epi mode with standard Gaussian detection - the backscattered light was recombined with the light propagating in reference arm and coupled to the spectrometer (diffraction grating: 1200 lpmm, Wasatch Photonics, camera: Basler Sprint, 140kHz, 2048 pixels). Insertion of cold mirror between objective lens and tube lens allowed for coupling remaining channels operating in visible region - brighfield microscopic system and the one delivering clot-inducing laser light from the laser diode (central wavelength: $520 \mathrm{~nm}$, Thorlabs). The laser diode spot position was adjusted by the set of deflecting mirrors (not included in Fig. 1 for clarity). Brightfield preview was used for precise navigation on the animal's brain area and for real-time preview of the clot formation. The exemplary images from this modality are presented in Fig. 3, where one can see the location of the inducing green laser diode light spot (3b) and the effect of clot formation procedure (3c). 


\subsection{Animal preparation}

\subsubsection{Cranial window implementation}

Young adult mice (6-10 weeks) were prepared for imaging by implanting a small imaging window centered over the barrel cortex. Mice were placed in stereotaxic frame and deeply anaesthetized with isoflurane (4\% for induction, $1.5-2 \%$ for surgery). Dexamethasone $(0.2 \mathrm{mg} / \mathrm{kg})$ and Carprofen $(5 \mathrm{mg} / \mathrm{kg})$ were administered subcutaneously to prevent swelling of the brain and inflammatory response. The body temperature of mice was monitored using a warming pad with anal probe. After removing the skin over the top of the skull a drop of lidocaine (1\%)-epinephryne (1:100000) solution were applied onto the periosteum to avoid excessive bleeding or pain. A circle about $4 \mathrm{~mm}$ diameter of the skull were removed and the dura was exposed. An optical chamber were then constructed by covering the intact dura with $1.2 \%$ low melting point agarose (Sigma) and $5 \mathrm{~mm}$ diameter cover glass glued with cyanoacrylate-based glue and sealed with dental acrylic.

\subsubsection{Photothrombotic stroke model implementation}

The actual OCM measurement session involving photothrombotic stroke model was executed after one week recovery from the cranial window installation. The animal was placed in stereotaxic frame with the worming pad and deeply anaesthetized with isoflurane ( $4 \%$ for induction, $1.5-2 \%$ for stoke procedure). The stereotaxic frame was positioned with the use of custom made 5-axis platform in the object space so the windowed part of the animal's brain was accessed by the extended focus of the imaging system. The whole 3D data sets were acquired at the rate limited by the speed of the CMOS camera, i.e. every 20 seconds. During the measurement procedure the position of the animal was not altered in any way. After 10 minutes the light sensitive dye rose bengal (Sigma) was administered intraperitoneally (100 $\mathrm{mg} / \mathrm{kg}$ of rose bengal in $100 \mu \mathrm{l}$ of $\mathrm{NaCl}_{2}$ ). 12 minutes later (time needed for the distribution of the agent over the whole body) 520 $\mathrm{nm}$ green lased diode beam was focused on bifurcation of middle cerebral artery (MCA) and stayed turned on for 20 minutes. The power of the diode light delivered to the sample was ca. $1 \mathrm{~mW}$. This level of power was proved in an independent measurement not to cause any functional nor structural changes when illuminated at Bengal-rose-free middle cerebral artery. After switching off the diode the measurement continued for 10 more minutes to observe further changes due to dynamic changes of location of the clots that had been produced.

\subsection{Scanning protocol and angiographic analysis}

The scanning protocol used to obtain the complete 3D data set was designed to acquire $300 \mathrm{~A}$-scans $\mathrm{x} 300 \mathrm{~B}$-scans over $1 \mathrm{~mm} \times 1 \mathrm{~mm}$ area. Each A-scan consisted of the 2048 points. The angiographic algorithms work with data acquired for particular point at the object in different time points. The separation between points in time space should be long enough to allow for decorrelation of the signal coming from the scatterers, here - the blood cells. It should be suited to the absolute scatterers velocity which in case of the vessels in mouse's brain spans up to $30 \mathrm{~mm} / \mathrm{s}$. If so, the measure of decorrelation becomes therefore a good contrast parameter for vascular network. B-scans were acquired 6 times before the slow axis scanner deflected the beam to next lateral position. This assured the time interval of ca. $5.5 \mathrm{~ms}$ between such oversampled B-scans. Thus the 4D data set for every measurement was acquired enabling pointwise speckle decorrelations analysis for angiographic imaging. The repetition time for every A-scan was $18 \mu \mathrm{s}$.

The analysis of the structure was performed with the standard procedures involving linearization spectrum in wavenumber space, numerical dispersion compensation and Fourier transform [9]. The oversampled data was simply averaged. For angiographic mapping the signal signal between oversampled B-scans was analyzed in terms of phase changes in time. For every single point of B-scan the variance of the local phase shifts [10] or the average of the difference between the total complex signal were calculated [11]. High resolution made procedure very sensitive for motion artifacts. The prealignment of the B-scans by adequate phase shifts of particular A-scans within the analyzed set [12] was needed which was based on the signal from manually indicated static structure. 


\section{RESULTS}

In Fig. 4 we present exemplary results of the OCM measurement of the mouse brain before and after production of the clot. Figure 4 shows structural and angiographic en face projections of the imaged region. The green point indicates the position of the green laser beam spot. One can see quantitative difference between both pairs of images before (top) and after (bottom) stroke induction, wherein the contrast of the blood vessels is much better on angiographic maps. The red arrows indicates the most prominent occlusions of the vessels. Interestingly, the vessel indicated by the left red arrow must have been occluded by the clot detached of the main clot produced at the very spot of green laser illumination and transported to the final destination by the main horizontal artery. One can also notice the significant reduction of the diameter of the main horizontal artery due to the changes of the internal pressure caused by occlusion of the other subartery originated in the bifurcation occluded by the main clot.
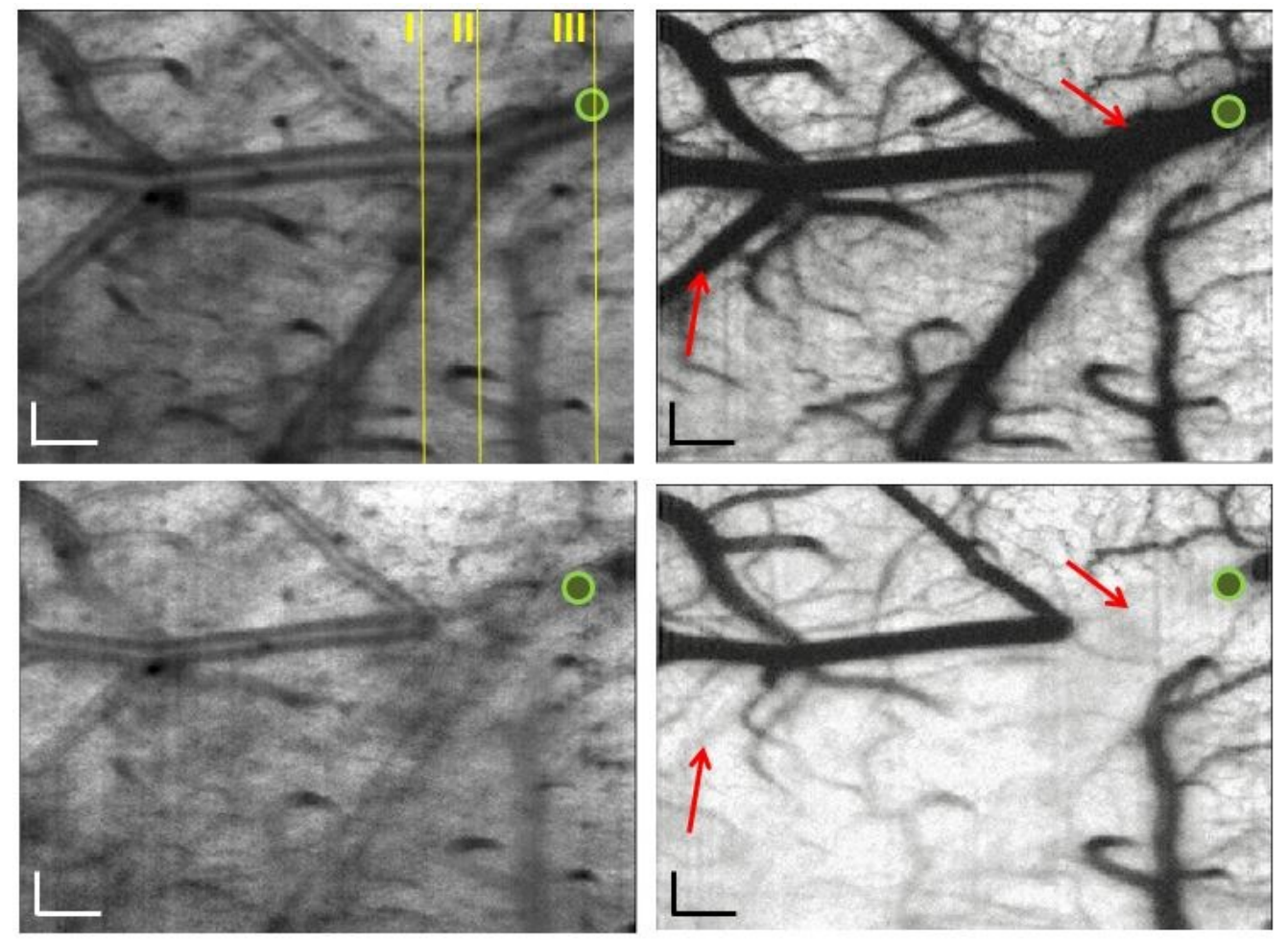

Fig. 4. Results of imaging of the mouse brain region before (top) and after (bottom) stroke induction procedure: structural (left) and angiographic (right) en face projections of the imaged region. Green spot indicates the focus of the green $520 \mathrm{~nm}$ laser diode light. Red arrows points at chosen vessels that were affected by occlusion.

In Fig. 5a we show the structural B-scans whose position in the data set is indicated by yellow vertical lines (I, II, III) in Fig. 4 before (upper set) and after (lower set) stroke induction procedure. One can recognize the cross-sections of the large vessels present in the map. Before occlusion in vessels are visible characteristic "butterfly" patterns indicating fast laminar blood flow. As a result of manifestation of the main clot the vessels in position I seems to got empty due to outflow of the blood. In bifurcation of the main artery (position II) and the green laser spot position (position III) one can recognize the appearance of scattering static medium which is identified as clot. Nevertheless, the main horizontal artery seems not to be closed completely and keeps supplying with the blood further parts of the vascular system. Corresponding phase variance maps (Fig. 5b) report on the actual dynamics within the vessels identified in Fig. 5a. For example it shows that the one of the vessels visible in (I) is actually not empty and contains flowing contents, nevertheless at clearly decreased rate. 
a)
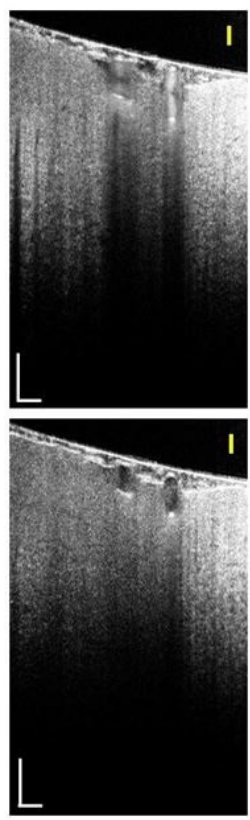
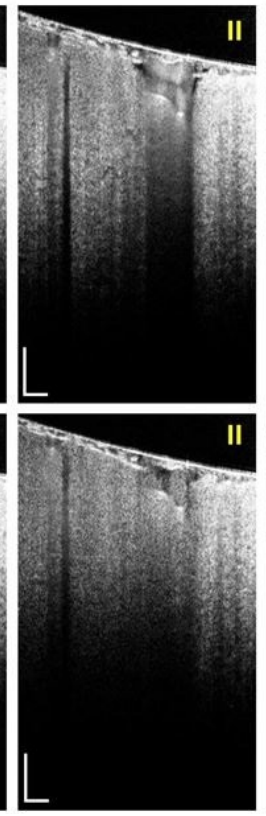

I
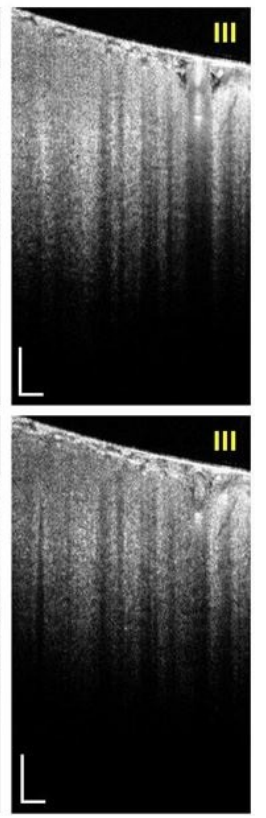

b)
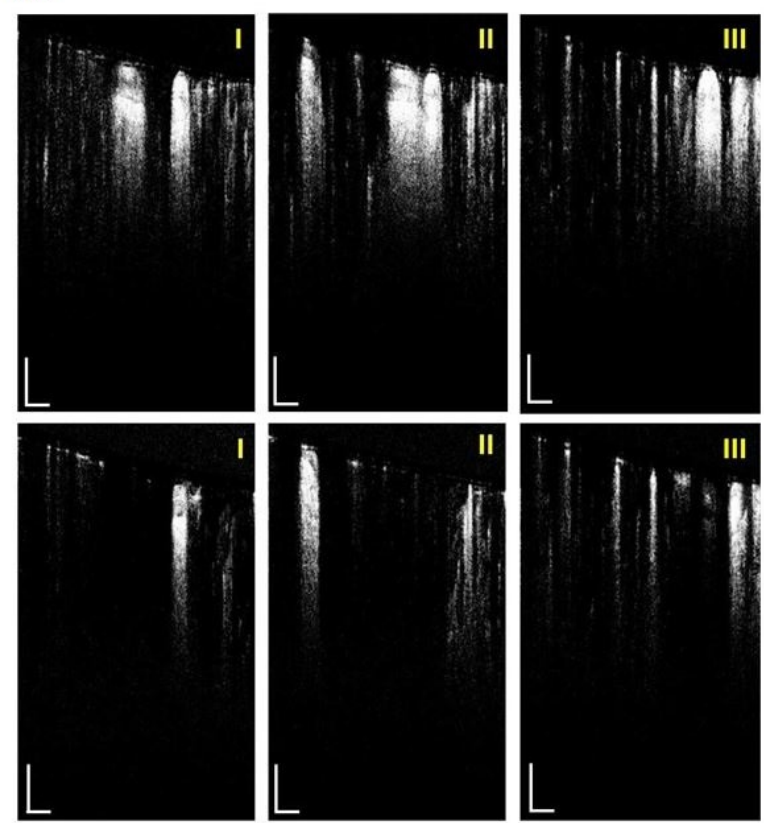

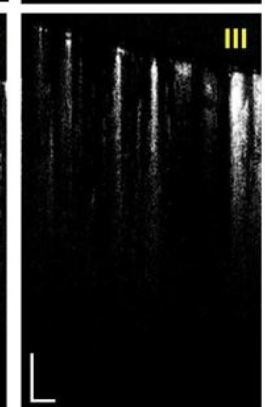

Fig. 5. Set a) shows structural B-scans and set b) shows phase variance B-scans corresponding to the cross-sections indicated by the yellow lines in Fig. 4 before (top) and after (bottom) induction of occlusions. Scale bars: $100 \mu \mathrm{m}$.

\section{DISCUSSION AND CONCLUSIONS}

The proposed combination of OCM monitoring and photothrombosis stroke induction procedure opens new possibilities of systematic investigation of the stroke dynamics. Localized occlusion is not a new idea, however obtained by optical means, as hereby presented, shows many important advantages over mechanical procedures like filament main cerebral artery ligature. The most important seems to be the fact, that in case in the photoactive induction of the stroke the continuous in situ 3D observation of the development of the stroke is possible in time over significantly large area of a brain containing main cerebral artery. The inherent property of the method is that the complete occlusion occurs in random moment, if at all. It is dependent on the activating light power, properties of the light beam, local concentration of the photoactive dye and properties of blood itself. However, the random character of the process brings it closer to the mechanism of the real ischemic stroke in humans and therefore makes it and attractive field of experimentation for neurologists. Another advantage is that the method allows for occluding any vessel in the field of view by unrestricted shift of the activating beam. The possibility of control of the diameter of the focal spot from single micrometers to hundreds of micrometers provides possibility to occlude single vessels of any diameter, in particular capillaries too small to perform mechanical filament fastening on them. If required, one can also illuminate the larger area of the brain, thus corrupting by clots vessels present within it. It can be helpful when stroke-related penumbra of particular region of the brain is the issue of interest.

OCM monitoring of the changes in brain is effectively enhanced by the use of Bessel beam providing extended focus. Requirements of the in vivo measurements imposed on the technique in terms of speed exclude the possibility of mechanical axial scanning of the brain to enhance the signal from deeper regions of the cortex. Presented solution allows for extending focus to ca. $500 \mu \mathrm{m}$ which is enough to penetrate dura and reach II/III region of the neocortex end thereby to cover the region most supplied by the blood vessels. An important limitation of the used optical configuration is the Gaussian detection which significantly limits the ability to collect the light from above and below the center of the focus. The attempt to remove this problem will be the aim for further work. It should allow for effective systematic real-time research on the development of the stroke and control of the affects of undertaken recovery treatment. 


\section{ACKNOWLEDGEMENTS}

This project was financed by National Centre for Research and Development Grant No. PBS1/A9/20/2013. Maciej Wojtkowski acknowledges National Science Centre project "Maestro" (decision no. DEC-2011/02/A/ST2/00302). Maciej Szkulmowski and Szymon Tamborski acknowledge „Iuventus Plus” programme for years 2015-2016 (grant IP2014 015973).

\section{REFERENCES}

1. A. G. Thrift, D. A. Cadilhac, T. Thayabaranathan, G. Howard, V. J. Howard, P. M. Rothwell, and G. A. Donnan, "Global stroke statistics," Int J Stroke 9, 6-18 (2014).

2. $\quad$ P. Mergenthaler and A. Meisel, "Do stroke models model stroke?," Disease Models and Mechanisms 5, 718725 (2012).

3. S. P. Chong, C. W. Merkle, D. F. Cooke, T. Zhang, H. Radhakrishnan, L. Krubitzer, and V. J. Srinivasan, "Noninvasive, in vivo imaging of subcortical mouse brain regions with 1.7 mum optical coherence tomography," Opt Lett 40, 4911-4914 (2015).

4. U. Baran and R. K. Wang, "Review of optical coherence tomography based angiography in neuroscience," NEUROW 3, 010902-010902 (2016).

5. R. A. Leitgeb, M. Villiger, A. H. Bachmann, L. Steinmann, and T. Lasser, "Extended focus depth for Fourier domain optical coherence microscopy," Opt. Lett. 31, 2450-2452 (2006).

6. A. Bouwens, T. Bolmont, D. Szlag, C. Berclaz, and T. Lasser, "Quantitative cerebral blood flow imaging with extended-focus optical coherence microscopy," Opt. Lett. 39, 37-40 (2014).

7. A. Schmidt, M. Hoppen, J.-K. Strecker, K. Diederich, W.-R. Schäbitz, M. Schilling, and J. Minnerup, "Photochemically induced ischemic stroke in rats," Experimental \& Translational Stroke Medicine 4, 13-13 (2012).

8. $\quad$ Y. Wang-Fischer, Manual of stroke models in rats (Boca Raton: CRC Press, 2009).

9. Optical Coherence Tomography: Technology and Applications, 2 ed. (Springer International Publishing, 2015).

10. J. Fingler, R. J. Zawadzki, J. S. Werner, D. Schwartz, and S. E. Fraser, "Volumetric microvascular imaging of human retina using optical coherence tomography with a novel motion contrast technique," Opt Express 17, 22190-22200 (2009).

11. V. J. Srinivasan, J. Y. Jiang, M. A. Yaseen, H. Radhakrishnan, W. Wu, S. Barry, A. E. Cable, and D. A. Boas, "Rapid volumetric angiography of cortical microvasculature with optical coherence tomography," Opt. Lett. 35, 43-45 (2010).

12. J. Lee, V. Srinivasan, H. Radhakrishnan, and D. A. Boas, "Motion correction for phase-resolved dynamic optical coherence tomography imaging of rodent cerebral cortex," Opt. Express 19, 21258-21270 (2011). 